\title{
The association between water kilomasers and compact radio sources in the starburst galaxy NGC 2146
}

\author{
A. Tarchi ${ }^{1,2}$, C. Henkel ${ }^{1}$, A. B. Peck ${ }^{1,3}$, and K. M. Menten ${ }^{1}$ \\ 1 Max-Planck-Institut für Radioastronomie, Auf dem Hügel 69, 53121 Bonn, Germany \\ 2 Istituto di Radioastronomia, CNR, via Gobetti 101, 40129 Bologna, Italy \\ 3 Harvard-Smithsonian Center for Astrophysics, 60 Garden St., Cambridge MA 02138, USA
}

Received 26 March 2002 / Accepted 16 May 2002

\begin{abstract}
We report the detection of $22 \mathrm{GHz}$ water vapor emission toward the starburst galaxy NGC 2146, made using the Effelsberg 100-m telescope. Interferometric observations with the Very Large Array (VLA) show that a part of the emission originates from two prominent sites of star formation that are associated with compact radio continuum sources, likely ultra-compact HII regions. It is concluded that the emission arises from the most luminous and distant $\mathrm{H}_{2} \mathrm{O}$ "kilomasers" detected so far. Our data increase the number of water maser detections in northern galaxies $\left(\mathrm{Dec}>-30^{\circ}\right)$ with IRAS point source fluxes $S_{100 \mu \mathrm{m}}>50 \mathrm{Jy}$ to $18 \%$.
\end{abstract}

Key words. galaxies: individual: NGC 2146 - galaxies: starburst - galaxies: ISM - masers - radio lines: ISM radio lines: galaxies

\section{Introduction}

Interferometric studies of extragalactic water masers indicate that $\mathrm{H}_{2} \mathrm{O}$ emission can arise in different environments. The weakest masers (with isotropic luminosities $\left.L_{\mathrm{H}_{2} \mathrm{O}}<0.1 L_{\odot}\right)$ are associated with prominent sites of star formation (e.g. in the LMC and SMC: Scalise \& Braz 1982; IC 342: Tarchi et al. 2002), while the strongest ones (defined as "megamasers"; $L_{\mathrm{H}_{2} \mathrm{O}}>20 L_{\odot}$ ) are related to nuclear activity in the host galaxy, associated either with accretion disks (e.g. NGC 4258: Greenhill et al. 1995) or with an interaction between the nuclear jet and molecular clouds (e.g. NGC 1052: Claussen et al. 1998; Mkn 348: Peck et al. 2001). In the intermediate range of water maser luminosities $\left(0.1 L_{\odot}<L_{\mathrm{H}_{2} \mathrm{O}}<20 L_{\odot}\right.$; the so called "kilomasers" $\left.{ }^{1}\right)$ there have been five extragalactic detections so far: M 33 [IC 133] (Churchwell et al. 1977), M 82 (Claussen et al. 1984), IC 10 (Henkel et al. 1986), and NGC 253 and $\mathrm{M} 51$ (Ho et al. 1987). Only the $\mathrm{H}_{2} \mathrm{O}$ maser associated with M 51 (Hagiwara et al. 2001) has a nuclear origin, while most of the others are known to be associated with star-forming regions. Detecting new kilomasers and obtaining accurate positions is essential to search for the numerous population of nuclear kilomasers proposed

Send offprint requests to: A. Tarchi,

e-mail: atarchi@ira.bo.cnr.it

1 For a reference introducing this nomenclature, see e.g. Hagiwara et al. (2001). by Ho et al. (1987), to identify regions of vigorous star formation in the outer disks, and to find targets that allow the determination of proper motions.

In this paper we report on the detection of the most luminous and most distant $\mathrm{H}_{2} \mathrm{O}$ kilomasers to date, and determine their positions with subarcsec accuracy toward the starburst galaxy NGC 2146.

\section{The observation and image processing}

Effelsberg We observed NGC 2146 in the $6_{16}-5_{23}$ transition of $\mathrm{H}_{2} \mathrm{O}$ (rest frequency: $22.23508 \mathrm{GHz}$ ) with the 100-m telescope of the MPIfR at Effelsberg ${ }^{2}$ on April 6 and December 22, 2000. The beam width was $40^{\prime \prime}$. The observations were made in a dual beam switching mode with a beam throw of $2^{\prime}$ and a switching frequency of $\sim 1 \mathrm{~Hz}$. The system temperature, including atmospheric contributions, was $\sim 200 \mathrm{~K}$ (Apr. 2000) and $\sim 80 \mathrm{~K}$ (Dec. 2000) on a main beam temperature scale. Flux calibration was obtained by measuring $\mathrm{W} 3(\mathrm{OH})$ (3.2 Jy). Gain variations of the telescope as a function of elevation were taken into account. The pointing accuracy was always better than $10^{\prime \prime}$.

\footnotetext{
2 The 100-m telescope at Effelsberg is operated by the MaxPlanck-Institut für Radioastronomie (MPIfR) on behalf of the Max-Planck-Gesellschaft (MPG).
} 
VLA NGC 2146 was observed on June 24, 2001 with the Very Large Array $^{3}$ (VLA) in its CnB configuration. We observed with two $25 \mathrm{MHz}$ IFs centered on the two maser features shown in Fig. 1. The source 0538+498 (1.93 Jy) was used as flux calibrator. The point source $0718+793$ was used for phase and bandpass calibration. The two IFs were calibrated separately and then "glued" together. The data were Fourier-transformed using natural weighting to create a $2048 \times 2048 \times 24$ data cube. The radio continuum was subtracted using the AIPS task UVLSF. This task fits a straight line to the real and imaginary parts of selected channels and subtracts the fitted baseline from the spectrum, optionally flagging data having excess residuals. In addition, it provides the fit continuum as a UV data set, which has been used to create the map shown in Fig. 2. The restoring beam is $0.9 \times 0.0^{\prime \prime} 6$ and the rms noise per channel is $0.6 \mathrm{mJy}$, slightly higher than the expected thermal noise. This is likely caused by a cloudy sky during the observations ${ }^{4}$.

\section{Results}

Figure 1 shows two $22 \mathrm{GHz} \mathrm{H}_{2} \mathrm{O}$ features, separated by $\sim 200 \mathrm{~km} \mathrm{~s}^{-1}$, at velocities almost equidistant from the systemic velocity of NGC 2146 (see also Table 1 ). The line width of each feature $\left(\sim 65 \mathrm{~km} \mathrm{~s}^{-1}\right)$ is larger than that of most other galactic or extragalactic maser components. The total isotropic luminosity of the emission is $\sim 8 L_{\odot}$ (see Table 1; $D=14.5 \mathrm{Mpc}$; Benvenuti et al. 1975). No other component was seen at velocities $380<V_{\mathrm{LSR}}<$ $1450 \mathrm{~km} \mathrm{~s}^{-1}$ (channel spacing: $4.2 \mathrm{~km} \mathrm{~s}^{-1} ; 5 \sigma$ noise level: $15 \mathrm{mJy})$.

Figure 2 shows the $22 \mathrm{GHz}$ VLA continuum map (central panel) obtained from the line-free channels as explained in Sect. 2 and the two spectra (small panels) of the maser emitting regions. Some compact radio sources are coincident with those detected at $5 \mathrm{GHz}$ by Tarchi et al. (2000; hereafter TNG), the positions of which are depicted by crosses. Differences between the map shown here and the $5 \mathrm{GHz}$ map of TNG are attributable to the lower sensitivity and coarser positional accuracy of the former, and to the nonthermal nature of some sources (i.e. their radio flux density decreases with increasing frequencies).

The water vapor emission seen by the VLA arises from two regions. The first one, at a position of $\alpha_{2000}=$ $06^{\mathrm{h}} 18^{\mathrm{m}} 36.64$ and $\delta_{2000}=78^{\circ} 21^{\prime} 27^{\prime \prime} \cdot 7$, is resolved and forms an extended structure with dimensions of $\sim 2^{\prime \prime} \times 1.5^{\prime \prime}$ $(140 \times 105 \mathrm{pc}$; Fig. 3a). The second region is located at $\alpha_{2000}=06^{\mathrm{h}} 18^{\mathrm{m}} 38^{\mathrm{s}} .63$ and $\delta_{2000}=78^{\circ} 21^{\prime} 24^{\prime \prime} .0$ and is only slightly resolved with dimensions of $\sim 1^{\prime \prime} \times 0.7^{\prime \prime}(70 \times 50 \mathrm{pc}$; Fig. 3b). Peak flux densities and restoring VLA beam size infer lower limits to the peak brightness temperature of only $\sim 10 \mathrm{~K}$ for the two $\mathrm{H}_{2} \mathrm{O}$ lines. Since, however,

3 The National Radio Astronomy Observatory is a facility of the National Science Foundation operated under cooperative agreement by Associated Universities, Inc.

${ }^{4}$ Sky coverage of $85 \%$ with clouds of cumuloform type.

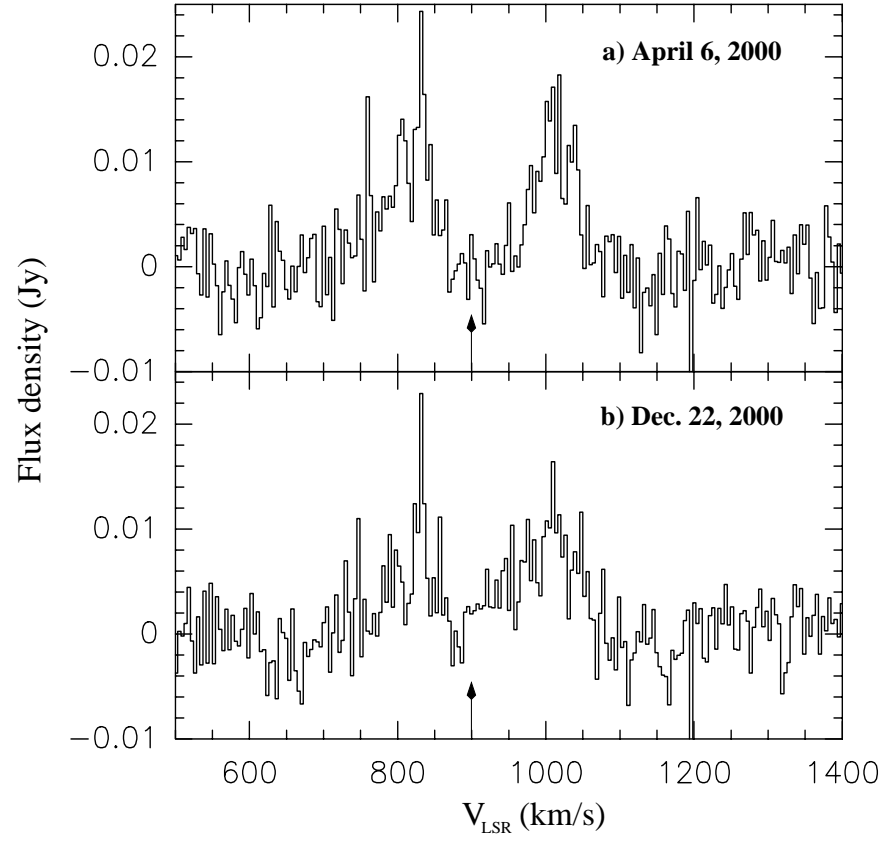

Fig. 1. Water maser features in NGC 2146 observed with Effelsberg on a) April 6, 2000, and b) December 22, 2000. Arrows mark the systemic velocity $\left(V_{\mathrm{LSR}}=900 \mathrm{~km} \mathrm{~s}^{-1}\right.$; De Vaucouleurs et al. 1991).

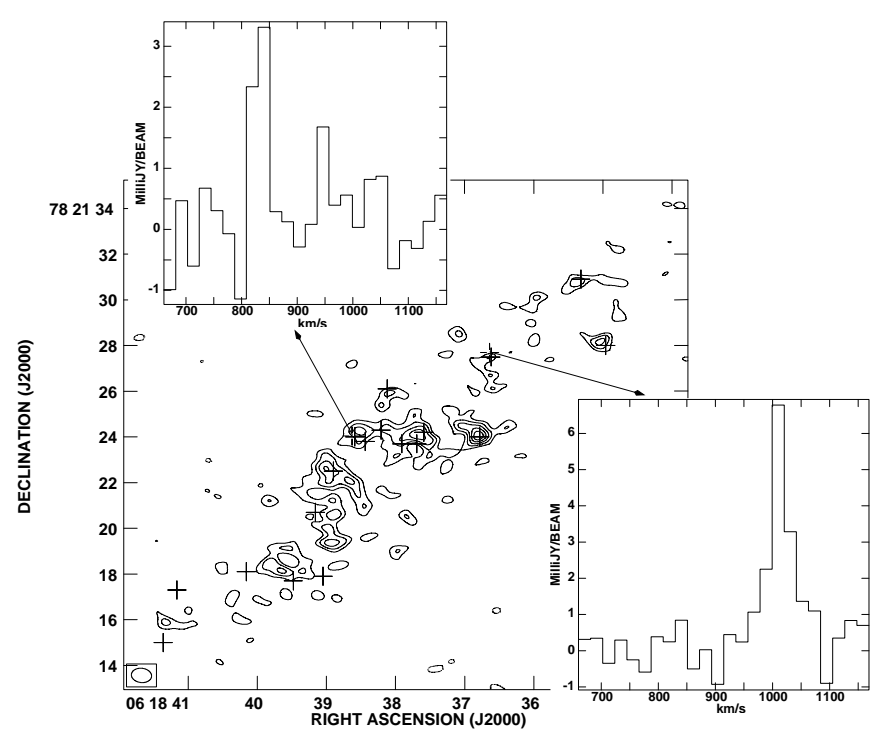

Fig. 2. $22 \mathrm{GHz}$ continuum VLA contour plot of NGC 2146 (main panel) with the spectra of the two maser spots (small panels). The contour levels are $-2.5,2.5,3.5,4.5,5.5,6.5,7.5 \sigma$ $\left(1 \sigma=0.2 \mathrm{mJy}\right.$ beam $\left.^{-1}\right)$. Crosses indicate the positions of the 18 compact radio sources (supernova remnants or HII regions) detected by TNG. The two crosses at the bases of the arrows mark the $\mathrm{H}_{2} \mathrm{O}$ maser spots. The synthesized beam of $0.0^{\prime \prime} 9 \times 0^{\prime \prime} 6$ is shown in the lower-left corner.

the $22 \mathrm{GHz}$ line is known to appear in all well studied sources as a maser, maser emission is also inferred in the following discussion for NGC 2146.

The maser positions coincide with two $(36.6+27.5$ and $38.6+24.0)$ of the eighteen continuum sources 
Table 1. $\mathrm{H}_{2} \mathrm{O}$ in NGC 2146: observational details and parameters of the two maser line components.

\begin{tabular}{lcccccccc}
\hline \hline Obs. Date & Tel. & $\begin{array}{c}T_{\text {int }}^{\mathrm{a})} \\
(\text { hours })\end{array}$ & $\begin{array}{c}\text { BW } \\
(\mathrm{MHz})\end{array}$ & $\begin{array}{c}\text { Vel. Res. } \\
\left(\mathrm{km} \mathrm{s}^{-1}\right)\end{array}$ & $\begin{array}{c}S_{\text {peak }}^{\mathrm{b})} \\
(\mathrm{mJy})\end{array}$ & $\begin{array}{c}V_{\mathrm{LSR}}^{\mathrm{c}} \\
\left(\mathrm{km} \mathrm{s}^{-1}\right)\end{array}$ & $\begin{array}{c}\Delta V_{1 / 2} \\
\left(\mathrm{~km} \mathrm{~s}^{-1}\right)\end{array}$ & $\begin{array}{c}\text { Luminosities }^{\mathrm{d})} \\
L \odot\end{array}$ \\
\hline Apr. 2000 & EFF & 9 & 80 & 4.2 & 13 & $820(3)$ & $70(9)$ & 4.3 \\
& & & & & 13 & $1010(3)$ & $60(6)$ & 4.0 \\
Dec. 2000 & EFF & 2 & \multirow{2}{*}{80} & 4.2 & 10 & $825(6)$ & $64(19)$ & 3.0 \\
& & & & & 10 & $1010(6)$ & $94(14)$ & 4.6 \\
Jun. 2001 & \multirow{2}{*}{ VLA } & \multirow{2}{*}{25} & 20 & 3.2 & $831(2)$ & $33(20)$ & 0.5 \\
& & & & & 6 & $1012(2)$ & $41(6)$ & 1.2 \\
\hline
\end{tabular}

a) $T_{\text {int }}$ includes Effelsberg $22 \mathrm{GHz} \mathrm{H}_{2} \mathrm{O}$ on- and off-source integration time or VLA integration time including overheads.

b) Peak fluxes calculated from integrated line intensities divided by linewidths (both obtained from Gaussian fits using the data reduction software package "CLASS").

c) $\mathrm{LSR}=$ Local Standard of Rest.

d) Obtained via $\left[L_{\mathrm{H}_{2} \mathrm{O}} / L_{\odot}\right]=0.023 \times\left[\int S \mathrm{~d} V / \mathrm{Jy} \mathrm{km} \mathrm{s}^{-1}\right] \times[D / \mathrm{Mpc}]^{2}, D=14.5 \mathrm{Mpc}$.
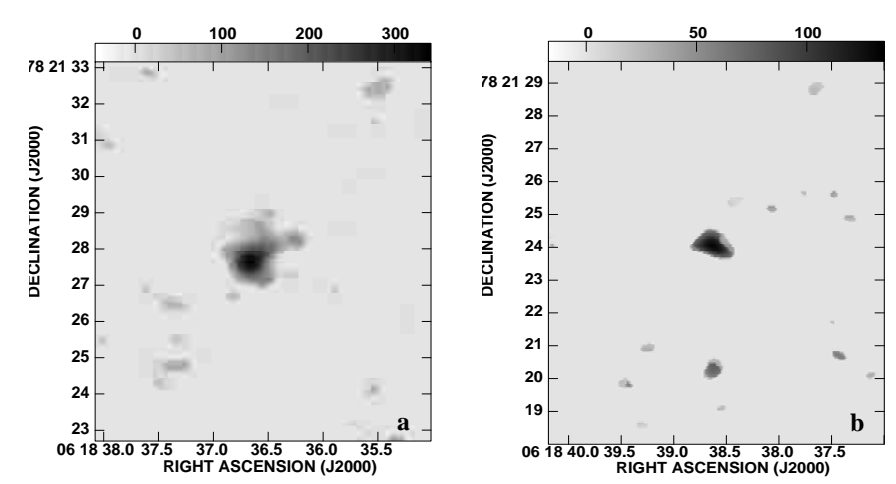

Fig. 3. Total integrated intensity (in $\mathrm{mJybeam}^{-1} \mathrm{~km} \mathrm{~s}^{-1}$ ) VLA grey-scale plots of the a) high-velocity and b) lowvelocity maser spots associated with the radio continuum sources $36.6+27.5$ and $38.6+24.0$, respectively. The synthesized beam is 0. . $9 \times 0.0^{\prime \prime} 6$.

detected by TNG, which are also visible (in one case only barely) in the continuum image of Fig. 2. The positional errors, dominated by statistical errors, have been estimated using the synthesized beam size divided by the signal-to-noise ratios ${ }^{5}$. The error on the continuum peak positions has been quadratically summed with that of the maser positions, giving a final value of 0 "' 16 for the positional uncertainty of both maser features. Within these uncertainties the continuum sources $(36.6+27.5$ and $38.6+24.0)$ and the maser spots are associated.

\section{Discussion}

The starburst galaxy NGC 2146 is characterized by large far infrared (FIR) and radio flux densities (e.g. Kronberg \& Biermann 1981; Smith et al. 1995) and by an outflow of hot gas along the minor axis driven by supernova explosions and stellar winds (Armus et al. 1995; Della Ceca et al. 1999; Greve et al. 2000). The star formation rate of NGC 2146, derived from its FIR flux density, is larger than that of the two starburst galaxies NGC 253 and M 82,

\footnotetext{
${ }^{5}$ For details on this derivation see e.g. Hagiwara et al. (2001).
}

which are also known to be $\mathrm{H}_{2} \mathrm{O}$ masers sources (Ho et al. 1987; Baudry \& Brouillet 1996).

\subsection{Nature of the water maser emission}

The nuclear position of NGC 2146 is near the compact radio source $37.6+24.2\left(\alpha_{2000}=06^{\mathrm{h}} 18^{\mathrm{m}} 37.58 ; \delta_{2000}=\right.$ $78^{\circ} 21^{\prime} 24^{\prime \prime} .2$; Tarchi et al. 2000). The two maser spots are at a projected galactocentric distance of $7^{\prime \prime}(490 \mathrm{pc})$ and are not located symmetrically on either side of the nucleus. The maser spots are not part of the innermost region of the galaxy.

Based on the spectral index $\left(S_{\nu} \propto \nu^{\alpha}\right)$, the radio continuum source associated with the north-western maser $(36.6+27.5)$ might be an optically thick compact HII region containing a number of massive stars (see TNG). A similar nature is also hypothesized for $38.6+24.0$ based on its detection at $5 \mathrm{GHz}$ but not at $1.6 \mathrm{GHz}$, implying a steep positive spectral index $\alpha$. The velocity of the two features is consistent with the velocity field of the central CO concentration, described as a warped molecular ring with an extent of $\sim 1 \mathrm{kpc}$ (Greve et al. 2000). The observed non-nuclear positions, the associated radio continuum sources, and the known connection between CO-emission and star formation indicate that the masers in NGC 2146 are related to sites of massive star formation and do not provide new evidence for the presence of a class of nuclear $\mathrm{H}_{2} \mathrm{O} 22 \mathrm{GHz}$ kilomasers that was postulated by Ho et al. (1987).

Integrated flux densities, peak intensities, and linewidths of the masers as derived from the VLA observations are smaller than the corresponding values from the single-dish detection (see Table 1). This could be due to time variability of the masers. The fact that the measured single-dish profiles are broad and look similar in April and December 2000 suggests, however, that each of the two main velocity components represents a large number of individual subcomponents whose intrinsic variations almost cancel out. This is further supported by the spatial extensions of the maser spots (see Fig. 3 and Sect. 3) that 
suggest that the emission is the result of an overlap of several unresolved masers of which only the strongest and most compact sources $(36.6+27.5$ and $38.6+24.0)$ have been detected. While the VLA data allow us to locate the two most prominent groups of maser features with modest signal-to-noise ratios, many additional features may be too weak to detect. Within this context it is interesting that continuum emission near the two sources mentioned is seen both in the 22 and $5 \mathrm{GHz}$ continuum maps (Fig. 2, TNG).

\subsection{Where to search for water maser emission?}

The new detection of water maser emission in NGC 2146 increases to $18 \%$ ( 8 out of 44 ) the detection rate of the complete sample of galaxies selected on the basis of their declination $\left(\delta>-30^{\circ}\right)$ and IRAS (Infrared Astronomy Satellite) fluxes $\left(S_{100 \mu \mathrm{m}}>50 \mathrm{Jy}\right.$; see Henkel et al. 1986; Tarchi et al. 2002). If we consider the subsample of galaxies having $S_{100 \mu \mathrm{m}}>100 \mathrm{Jy}$, which includes NGC 2146 , the detection rate is $\sim 30 \%$ ( 6 out of 19 ). Even taking into consideration that we are still dealing with small number statistics, the sample represents a unique case since typical searches for extragalactic water maser emission yield detection rates below (usually well below) 10\% (e.g. Henkel et al. 1984, 1998; Braatz et al. 1996; Greenhill et al. 2002). The high detection rate may be more due to the proximity of the galaxies surveyed rather than due to a physical relationship between maser and IRAS far-infrared emission (Greenhill 2001).

Within a radius of $20 \mathrm{Mpc}, 16$ external galaxies are known to emit $22 \mathrm{GHz} \mathrm{H}_{2} \mathrm{O}$ emission (marginal detections that are not included in our compilation were reported by Huchtmeier et al. 1978, 1988). There are seven megamasers with nuclear origin (NGC 1052, NGC 1068, NGC 1386, NGC 3079, NGC 4258, NGC 4945, Circinus; all classified as Sy 2 s and/or LINERs, and all spirals except the elliptical NGC 1052), 3 are low-luminosity "galactictype" masers (the LMC, the SMC, and IC 342), and the remaining six sources (M 33 [IC133], IC 10, NGC 253, NGC 2146, M 82, M 51) host kilomasers with average flux densities equal to or larger than the strongest galactic masers. Interferometric studies have shown that the masers in M 33 [IC133], IC 10, and M 82 (all spirals or irregulars) are associated with off-nuclear star-forming regions. Hagiwara et al. (2001) found that the $\mathrm{H}_{2} \mathrm{O}$ emission in M 51 (spiral, HII, Sey2.5) is nuclear in origin, possibly associated with the receding jet. For NGC 253 (spiral, starburst) interferometric studies are not yet published.

So far, the less luminous masers associated with star-forming regions have all been detected at distances $<4 \mathrm{Mpc}$, while all the luminous masers associated with nuclear activity, and hosted by AGNs, have been found at greater distances. The masers detected in NGC 2146 are the first with non-nuclear origin and $D>4 \mathrm{Mpc}$. These results point once more to the necessity of deep searches for $\mathrm{H}_{2} \mathrm{O}$ maser emission in galaxies with large IRAS flux densities. These have been shown to possess a particularly large number of detectable masers from star forming regions that might be excellent targets to determine not only radial velocities but also galactic proper motions and distances.

Acknowledgements. We wish to thank M. Claussen, Y. Hagiwara and E. Ros for useful comments and discussions. We are also endebted to the operators at the $100-\mathrm{m}$ telescope, and to M. Rupen and the NRAO analysts, for their cheerful assistance with the observations.

\section{References}

Armus, L., Heckman, T. M., Weaver, K. A., \& Lehnert, M. D. 1995, ApJ, 445, 666

Baudry, A., \& Brouillet, N. 1996, A\&A, 316, 188

Benvenuti, P., Capaccioli, M., \& D'Odorico, S. 1975, A\&A, 41, 91

Braatz, J. A., Wilson, A. S., \& Henkel, C. 1996, ApJS, 106, 51 Churchwell, E., Witzel, A., Pauliny-Toth, I., et al. 1977, A\&A, 54, 969

Claussen, M. J., Heiligman, G. M., \& Lo, K. Y. 1984, Nature, 310,298

Claussen, M. J., Diamond, P. J., Braatz, J. A., Wilson, A. S., \& Henkel, C. 1998, ApJ, 500, L129

Della Ceca, R., Griffiths, R. E., Heckman, T. M., Lehnert, M. D., \& Weaver, K. A. 1999, ApJ, 514, 772

De Vaucouleurs, G., De Vaucouleurs, A., Corwin, Jr., et al. 1991, Third Reference Catalogue of Bright Galaxies, Version 3.9

Greenhill, L. J. 2001, in Cosmic Masers proc. of IAU Symp., 206 (Mangaratiba, Brazil; March 2001) [astro-ph/0109419]

Greenhill, L. J., Jiang, D. R., Moran, J. M., et al. 1995, ApJ, 440,619

Greenhill, L. J., Ellingsen, S. P., Norris, R. P., et al. 2002, ApJ, 565,836

Greve, A., Neininger, N., Tarchi, A., \& Sievers, A. 2000, A\&A, 364, 409

Hagiwara, Y., Henkel, C., Menten, K., \& Nakai, N. 2001, ApJ, 560, L37

Henkel, C., Güsten, R., Wilson, T. L., et al. 1984, A\&A, 141, L1

Henkel, C., Wouterloot, J. G. A., \& Bally, J. 1986, A\&A, 155, 193

Henkel, C., Wang, Y. P., Falcke, H., Wilson, A. S., \& Braatz, J. A. 1998, A\&A, 335, 463

Ho, P. T. P., Martin, R. N., Henkel, C., \& Turner, J. L. 1987, ApJ, 320, 663

Huchtmeier, W. K., Witzel, A., Kühr, H., Pauliny-Toth, I. I., \& Roland, J. 1978, A\&A, 64, L 21

Huchtmeier, W. K., Eckart, A., \& Zensus, A. J. 1988, A\&A, 200, 26

Kronberg, P. P., \& Biermann, P. 1981, ApJ, 243, 89

Peck, A. B., Falcke, H., Henkel, C., \& Menten, K. M. 2001, The Central Kiloparsec of Starbursts and AGN, ed. J. H. Knapen, J. E. Beckman, I. Shlosman, \& T. J. Mahoney (San Francisco), ASP Conf. Proc., 249, 321

Scalise, E. Jr., \& Braz, M. A. 1982, AJ, 87, 528

Smith, B. J., Harvey, P. M., \& Lester, D. F. 1995, ApJ, 442, 610

Tarchi, A., Neininger, N., Greve, A., et al. 2000, A\&A, 358, 95 (TNG)

Tarchi, A., Henkel, C., Peck, A. B., \& Menten, K. M. 2002, A\&A, 385, 1049 